\title{
Caracterización de un modelo murino de enterocolitis necrotizante
}

\section{Characterization of a murine model of necrotizing enterocolitis}

\author{
DOI: $10.46932 / \operatorname{sfjdv2n5-034~}$
}

Received in: Oct 1st, 2021

Accepted in: Dec 30th, 2021

\author{
Oscar de Jesus Reyes Delgado \\ MD \\ Universidad Autónoma de Aguascalientes \\ Av. Universidad \#940, Ciudad universitaria C.P. 20100, Aguascalientes, Ags. México \\ E-mail: oscar_pxndx12@hotmail.com \\ Bibiana Moreno Carranza \\ P.h.D \\ Instituto de Neurobiología, Universidad Nacional Autónoma de México \\ Laboratorio A-15, Instituto de Neurobiología - UNAM, Boulevard Juriquilla 3001, Juriquilla, \\ Querétaro, México, C.P. 76230, Querétaro, México \\ E-mail: bibiana.moreno@yahoo.es
}

\section{RESUMEN}

Introducción: La enterocolitis necrotizante $(\mathrm{ECN})$ es la patología gastrointestinal de las más comunes y devastadoras en recién nacido con muy bajo peso al nacer (rango entre 500-1500g) y se caracteriza por inflamación y necrosis intestinal. Los objetivos de este estudio fueron desarrollar un modelo murino de ECN así como un modelo de sobreexpresión de proteínas en el intestino mediante la administración enteral mediante sonda de vectores lentivirales.

Métodos: Para el modelo de ECN se utilizaron cepas de ratón C57BL6 y CD1 a los cuales se les trató por 6 veces cada dos horas con una dosis de anoxia con CO2 al 100\% durante 10 o 7.5 minutos seguida una reoxigenación mediante hiperoxia al $95 \%$ por 5 minutos. Además, para activar el sistema inmune se administró LPS en las primeras dos dosis. Para la sobreexpresión de prolactina (PRL) en el intestino se administraron vectores lentivirales que sobreexpresan GFP (como control) o PRL por vía enteral a ratones CD1 en edades postnatales P2 y P3. Posteriormente se analizó la presencia de GFP y prolactina de las muestras de intestino mediante visualización por microscopia de fluorescencia y Western blot, respectivamente.

Resultados: Se obtuvo una mortalidad del $45 \%$ y una eficiencia de desarrollo de ECN entre los animales vivos del $100 \%$ en ratones CD1 de edad postnatal P1, en contraste con la mortalidad de $85 \%$ y la eficiencia de desarrollo de ECN entre los animales vivos del 0\% en ratones C57B16 de P1. En relación al modelo de sobreexpresión de proteínas en el intestino, se detectó GFP en el intestino de ratones administrados con $106 \mathrm{TU} / \mathrm{ml}$ vectores lentivirales para la sobreexpresión de GFP en el día P2 y evaluados 24 horas después. No se observó la sobreexpresión de PRL en el intestino de ratones administrados con 106 y 108 TU/ml vectores lentivirales para la sobreexpresión de PRL en los días P2 y P3 y evaluados 48 horas después.

Conclusión: El modelo de ECN en ratones CD1 de P1 tuvo una efectividad del $100 \%$ a pesar de una mortalidad elevada. Además, se logró estandarizar el método para la sobreexpresión de proteínas en el intestino de ratones en P2 24 horas después de la administración de vectores lentivirales por la via enteral. La determinación de sobreexpresión de PRL en el intestino no fue conclusiva.

Palabras clave: enterocolitis necrotizante, prolactina, inflamación, intestino delgado. 


\begin{abstract}
Background: Necrotizating enterocolitis (NEC) is one of the most common and devastating gastrointestinal disease in newborns with very low weight birth (range among 500 -1500 g). NEC is characterized by intestinal inflammation and necrosis. Our aims of this study were to develop a NEC murine model and a intestinal protein expression model by means of enteral administration of lentiviral vectors.

Method: For the NEC model were used C57BL6 and CD1 mice which were treated with anoxia with $100 \% \mathrm{CO} 2$ for 10 or 7.5 minutes followed by $95 \%$ O2 for 5 minutes. This treatment was repeated six times with a 2 hours interval. Moreover, to activate the immune system, LPS was administrated orally in the first two doses. For the overexpression of prolactin (PRL) in the intestine, lentiviral vectors that overexpress GFP (as a control) or PRL were administered by orally to CD1 mice at postnatal ages P2 and P3. Then, the presence of GFP and prolactin in the intestine samples was analyzed by fluorescence microscopy and Western blot, respectively.

Result: Mortality of $45 \%$ and a NEC development efficiency of $100 \%$ was obtained among live animals in CD1 mice of P1 postnatal age, in contrast to the mortality of $85 \%$ and development efficiency of $0 \%$ among live animals in C57BL6 mice of P1 age. In relation to the protein overexpression model in the intestine, GFP was detected in the mice gut administrated with 106 lentiviral vectors for the GFP overexpression on P2 evaluated 24 hours later. PRL overexpression was not observed in mice that received on day P1 postnatal 106 and $108 \mathrm{TU} / \mathrm{ml}$ of lentiviral vectors for the overexpression of PRL and evaluated on days P2 and P3.

Conclusion: NEC model had an effectiveness of 100\% in CD1 mice of 1 day of life, despite the high mortality. Moreover, a method for protein overexpression in the intestine was standardized. Lenviral vectors were orally administered 24 hours after birth and the expression of the protein was detected 24 hours later. Prolactin overexpression determination was not conclusive.
\end{abstract}

Keywords: necrotizing enterocolitis, prolactin, inflammation, small intestine.

\title{
1 INTRODUCCIÓN
}

La enterocolitis necrotizante $(\mathrm{ECN})$ se caracteriza por una inflamación y necrosis isquémica de la mucosa intestinal, e invasión de microorganismos formadores de gas dentro de la pared intestinal. (1) La ECN está entre una de las patologías gastrointestinales más comunes y devastadoras en neonatos. La incidencia de ECN está relacionada con partos prematuros, función intestinal inmadura, isquemia e hipoxia intestinal, colonización bacteriana anormal y factores genéticos. (2) Ha sido también una de las patologías más difíciles de erradicar, motivo por el cual es prioridad para investigación. El excesivo proceso inflamatorio iniciado por la alta inmunoreactividad intestinal extiende sistémicamente los efectos de la enfermedad, afectando principalmente a cerebro, con un riesgo del $25 \%$ para desarrollar microcefalia y retraso en el neurodesarrollo. La prevalencia de esta entidad es del 7\% entre los infantes con un peso al nacer entre los 500 y 1500 g y tiene un rango estimado de mortalidad de entre el 20 y 30\%. Los signos y síntomas iniciales más típicos en la enterocolitis necrotizante "clásica" son intolerancia a la vía oral, distensión abdominal y sangre en heces después del día 8 y 10 de edad. Los hallazgos patognomónicos en 
la radiografía abdominal son pneumatosis intestinal, presencia de gas en el sistema porta hepático o ambos. (3)

Existen tres áreas de descubrimiento científico las cuales son las más aceptadas para el avance del conocimiento de ECN en infantes prematuros. La primera habla de un mecanismo de isquemia e inflamación intestinal y el sistema gastrointestinal prematuro. En el intestino inmaduro ante una agresión como hipoxia, hipoperfusión, alimentación enteral con fórmula y la eventual colonización aberrante hacen más probable que un proceso isquémico ocurra acompañado de una respuesta inflamatoria temprana y exagerada. Todo ello lleva a una lesión epitelial, generando un mecanismo de retroalimentación positiva inflamatorio, liberando mediadores inflamatorios que exacerban la respuesta por el intestino inmaduro. Estas características del sistema gastrointestinal pretérmino lo hacen vulnerable a la isquemia intestinal, lesión hiperosmolar, invasión bacteriana y la subsecuente inflamación, las cuales están más probablemente implicadas en el desarrollo de NEC.

La segunda se relaciona con la alimentación por vía oral. Está identificado que la alimentación enteral que llega al lumen intestinal de los neonatos causa disrupción de la integridad de la mucosa, disminución del flujo sanguíneo y de la motilidad. Los nutrientes que no son absorbidos en el intestino delgado y grueso favorecen la proliferación bacteriana, la cual puede producir distensión, neumatosis intestinal y aumento de la presión intraabdominal que lleva a una mayor disminución del flujo sanguíneo. Por otra parte, la alimentación enteral con leche materna ha mostrado ser protectora contra NEC en aspectos nutricionales e inmunológicos.

Como tercera área se habla de una colonización aberrante. Antes del nacimiento el intestino es esencialmente estéril. Al nacer este es colonizado y bajo circunstancias ideales un sistema inmune de defensa intestinal se desarrolla. Los infantes alimentados con leche materna tienen una menor incidencia de NEC que aquellos alimentados con fórmula. Esto se explica porque la lactancia facilita la colonización de una balanceada y no patológica microbiota en el intestino que ayuda a prevenir sobrecrecimiento bacteriano. La colonización aberrante produce una inducción tóxico-alimentaria por patrones moleculares microbianos que alteran la barrera epitelial y desencadenan una cascada inflamatoria intestinal innata del sistema inmune. (4)

A nivel molecular también se han hecho avances en el conocimiento de la patología de la ECN. Se conoce que en la lámina propia del intestino de ratones y neonatos prematuros con NEC existe un descenso en la producción de células T reguladoras y un incremento en la producción células Th17 CD4+. La liberación de IL-17 por los Th17 CD4+ resulta en una lesión de la mucosa intestinal, demostrada por una alteración en las uniones estrechas, un aumento en la apoptosis y una reducción de la proliferación de los enterocitos. (5) Se observó que, en un modelo de artritis inflamatoria, la prolactina reduce niveles de 
proteína C reactiva y TNF-a, así como factores de transcripción y citocinas asociadas con las células Th17. (6) Estos datos indican el papel importante que podría estar jugando la prolactina en la maduración intestinal mediante la modulación de la respuesta inmune lo cual conllevaría a una reducción del riesgo de desarrollar NEC.

Los objetivos de este estudio son:

- establecer un modelo de NEC en ratones recién nacido basándonos en el protocolo reportado por Kyuwhan Jung et al con modificaciones, a saber, administración oral de LPS

- establecer el método de transducción intestinal a través de la inoculación enteral de vectores lentivirales para la sobreexpresión de prolactina (LV-PRL) o GFP como control (LV-GFP)

\section{MATERIALES Y MÉTODOS}

Modelo de ECN. Se utilizaron dos cepas de ratones: C57BL6 y CD1 en diferentes edades postnatales (P1, P2 y P3). Se les indujo hipoxia con $100 \%$ de $\mathrm{CO}_{2}$ durante 10 o 7.5 minutos, seguido de una reoxigenación con $95 \%$ de $\mathrm{O}_{2}$ por 5 min. Este proceso se repitió seis veces con un intervalo de 2 horas entre cada dosis de anoxia-reoxigenación. Posterior a la dosis se colocaron a las crías con su respectiva madre durante todo el intervalo de tiempo. Antes de las primeras dos dosis de anoxia-reoxigenación se administraron por vía enteral un volumen determinado de LPS en una concentración $2 \mu \mathrm{g} / \mu 1$ (Lipopolysaccharides, from Escheerichia coli, 055:B5, SIGMA). Posteriormente se extrajeron todos los tractos gastrointestinales de las crías, se aisló el íleon donde el terció proximal se preparó para una ulterior extracción de RNA y los dos tercios distales se consignaron para su procesamiento histológico.

Transducción de células intestinales tras la administración enteral mediante sonda de vectores lentivirales. Como control de la transducción de las células intestinales con los vectores lentivirales, iniciamos con la administración enteral mediante sonda de distintas concentraciones de LV-GFP $\left(10^{4}, 10^{6}\right.$ y $10^{8} \mathrm{TU} / \mathrm{ml}$ de vectores lentivirales y un grupo control tratados con PBS) a 8 ratones CD1 de P2. De dichos ratones se extrajeron muestras de tractos gastrointestinales para su posteriormente visualizaron mediante microscopia de fluorescencia. Se utilizaron 12 ratones CD1 en dos grupos de 6 ratones. En el primero la administración del vector lentiviral se realizó en P2 de los cuales dos se usaron como controles, a dos se administró una dosis via enteral $1 \times 10^{6} \mathrm{TU} / \mathrm{ml}$ de LV-PRL, y a los últimos dos se administró $1 \times 10^{8}$ TU/ml de LV-PRL. Para el segundo grupo se realizó el mismo procedimiento pero en P3. Posteriormente se realizó un análisis con Western blot (acrilamida 13\%, 10 pozos de $1.5 \mathrm{~mm}$ ) con $100 \mathrm{ng}$ de cada una de las muestras de intestino tratadas con LV-PRL y de muestras control sin tratamiento. Mediante anticuerpo anti-prolactina se determinó la presencia de ella y con el anticuerpo anti-tubulina se estandarizó nuestro método. 


\section{RESULTADOS}

Modelo ECN con cepa CD1. Seis crías (de 11 iniciales) de edad postnatal P1 sobrevivieron al procedimiento de inducción de ECN y presentaron signos macroscópicos de ECN (figura 1A y 2B). Por otra parte, de la inducción de ECN en edad postnatal P2, un 50\% de las crías murieron (3 de 6) y de las que sobrevivieron solo 1 presentó características indeterminadas y el resto no mostraron ningún dato anormal en el tracto GI. Finalmente, 6 crías de P3 fueron tratadas con el procedimiento de inducción de NEC sin mostrar mortalidad ni signos de NEC (tabla 1). Estos datos parecen indicar que el porcentaje de animales que desarrolla signos de ECN disminuye con la edad. Sin embargo, queda por evaluar si dosis de LPS administrada (que fue en todos la misma) pudiera enmascarar estos resultados y hacer necesario ajustarla en relación al peso en cada edad postnatal.

Modelo de ECN con la cepa C57BL6. Es de notar que en las crías de esta cepa se ajustó la dosis de LPS por peso del animal a $2 \mathrm{ug} / \mathrm{kg}$ de peso corporal. En los animales de P1 no se visualizó macroscópicamente ningún caso de ECN en ninguna de las edades utilizadas (tabla 1). Sin embargo, dos crías de esta edad postnatal mostraron datos de distensión de asas intestinales, hemorragias petequiales en todo el tracto gastrointestinal (GI) y marcada vascularización de peritoneo, por lo que se incluyeron en el grupo de indeterminados. El resto de las crías no mostraron cambios u anormalidades en el tracto GI. Cabe mencionar que solo un ratón de esta edad soportó hasta sexta dosis de anoxia-reoxigenación. Dado estos resultados de elevada mortalidad en la edad postnatal P1 se decidió disminuir el tiempo de anoxia a 7.5 min para las siguientes camadas. En las edades postnatales P2 y P3 con 7.5 minutos de anoxia y dosis de LPS de 2 ug/kg de peso corporal, no se logró obtener ECN la mortalidad fue aun muy elevada, del 83\%. Estos datos indican que la cepa C57BL6 es mas sensible al protocolo de indución de ECN mostrando una mortalidad mas elevada que los animales CD1 en todas las edades.

Sobreexpresión de proteínas en el intestino. Tras la administración enteral de LV-GFP se logró detectar a la proteína GFP mediante visualización de la fluorescencia en las microvellosidades intestinales (figura 3B) de ratones de edad postnatal P2 administrados con $10^{6} \mathrm{TU} / \mathrm{ml}$ de virus de GFP . Para aquellas crías tratadas con el LV-PRL no se logró determinar la presencia de prolactina en ninguna de las concentraciones utilizadas (figura $2 \mathrm{~A}$ y B). 
Tabla 1. Modelo de ECN con las cepas CD1 y C57.

\begin{tabular}{lllllll} 
Cepa & \multicolumn{2}{c}{ CD1 } & \multicolumn{2}{c}{ C57 } \\
\hline Camada & 1 & \multicolumn{2}{c}{2} & 1 & \multicolumn{2}{c}{2} \\
\cline { 3 - 4 } Edad & $\mathrm{P} 1$ & $\mathrm{P} 2$ & $\mathrm{P} 3$ & $\mathrm{P} 1$ & $\mathrm{P} 2$ & $\mathrm{P} 3$ \\
\# Crías & 11 & 6 & 6 & 7 & 6 & 6 \\
ECN & 6 & 1 & 0 & 0 & 0 & 0 \\
Indeterminado & 0 & 1 & 0 & 2 & 0 & 0 \\
Muertos & 5 & 3 & 0 & 6 & 5 & 5 \\
Normal & 5 & 4 & 6 & 5 & 6 & 6 \\
Min de CO $\mathrm{CO}_{2}$ & 10 & 10 & 10 & 10 & 7,5 & 7,5 \\
Dosis de LPS & $5 \mu \mathrm{l}$ & $5 \mu 1$ & $5 \mu \mathrm{l}$ & $5 \mu 1$ & $7 \mu 1$ & $11.4 \mu 1$ \\
\hline
\end{tabular}

Figura 1. Muestras de tractos gastrointestinales (desde estómago hasta íleon) con ECN de la primera camada de ratones CD1 (A) y muestra de tracto GI con ECN de la segunda camada de CD1 (B).

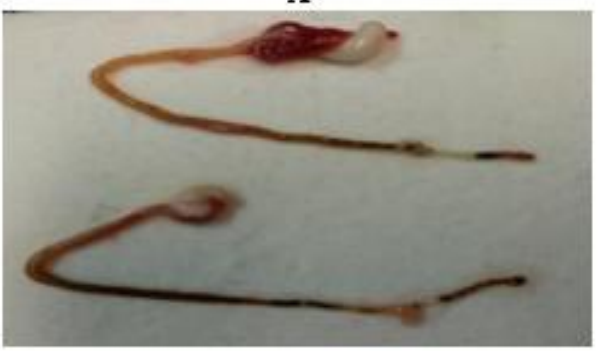

B

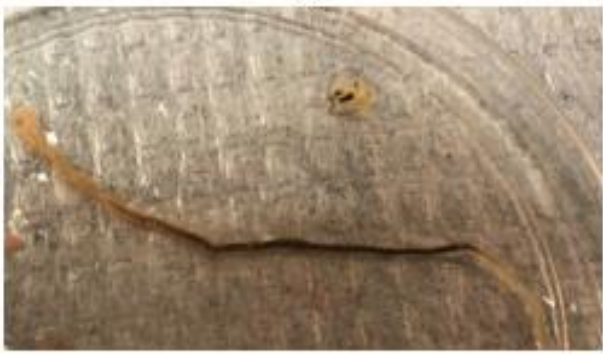

Figura 2. Blot de muestras de intestino tratadas a diferentes concentraciones $\left(10^{6} \mathrm{y} 10^{8} \mathrm{TU} / \mathrm{ml}\right)$ de virus de prolactina (A) en distintos días postnatales (P2 y P3) y un segundo blot para estandarización (B), con muestras de intestinos a las mismas concentraciones de prolactina, mediante un anticuerpo anti-tubulina. PRL: prolactina.

A

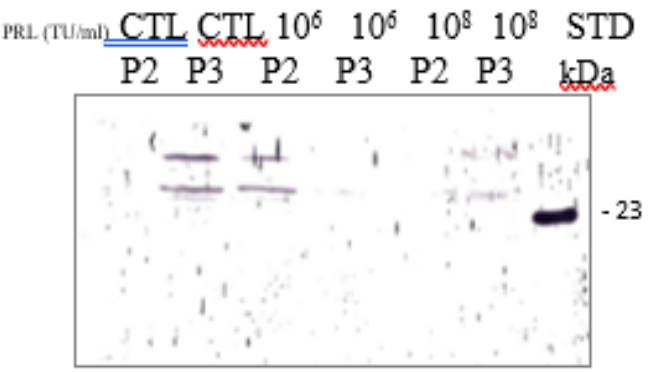

B

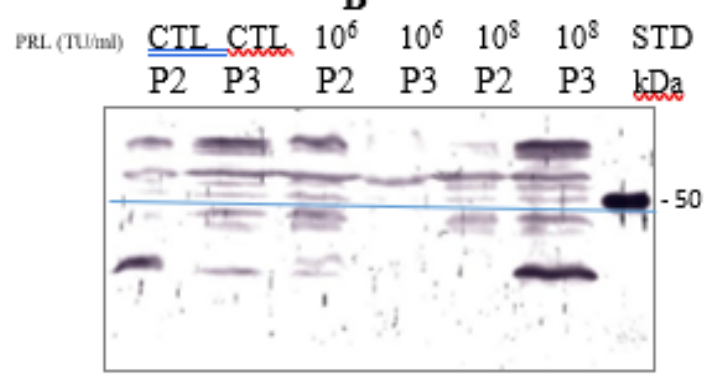

Figura 3. Imágenes de microscopía de fluorescencia de especímenes de intestinos, una control tratada con PBS (A), donde el recuadro de la parte inferior derecha indica un aumento de la parte central de la imagen que señala las vellosidades intestinales, $\mathrm{Y}$ en la otra imagen se muestra un fragmento de intestino tratado con $10^{6} \mathrm{TU} / \mathrm{ml}$ de virus GFP (green flourescent protein) (B), en la esquina inferior derecha se observa un aumento de la parte central de la imagen que señala una microvellosidad intestinal con expresión de GFP detectable a microcoscopia de fluorescencia (punta de flecha).

A

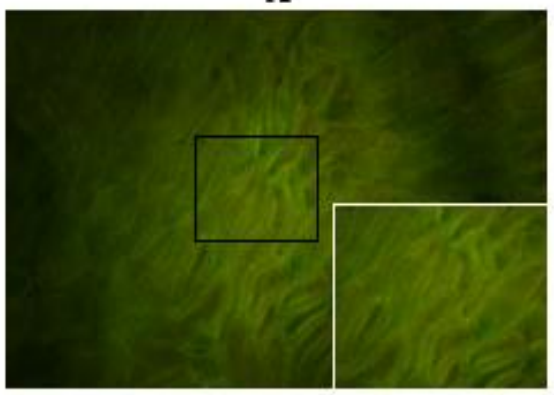

B

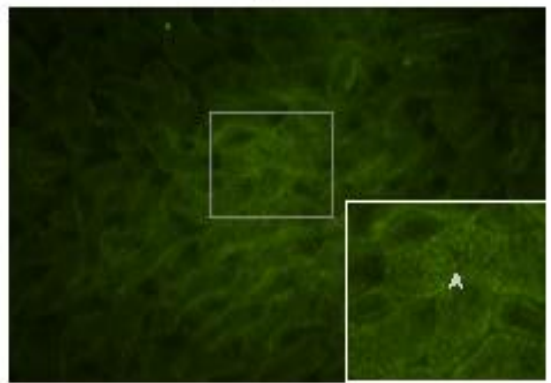




\section{CONCLUSIÓN}

El modelo de ECN modificado de Kyuwhan Jung et al. tuvo una efectividad del 100\%, al menos, en la primera camada tratada con este modelo. Se conoce que la administración de LPS activa la respuesta inflamatoria ya esta molécula es reconocida por los PRRs (receptores de reconocimiento de patrones) de tipo TLR4 y se activa la casada de señalización que lleva a incrementar los niveles de factor activador de plaquetas y por lo tanto, agravar la respuesta inflamatoria misma de la anoxia-reoxigenación (8).

Se logró estandarizar el método de transducción de células intestinales tras la administración enteral de vectores lentivirales que codifican para GFP pero de aquellos que codifican para PRL. Este método es conveniente ya que es una herramienta de asegurar una generación y liberación contínua de proteínas en la luz intestinal aunada a un riego minimizado de activación de respuesta $\mathrm{T}$ citotóxica-virus específica (9), con lo que se evita un daño en el tejido en estudio. 


\section{BIBLIOGRAFÍA}

1. Chariton Moschopoulos, Panagiotis Kratimenos, Ioannis Koutroulis, Bhairav V. Shah, Anja Mowes, and Vineet Bhandari, "The Neurodevelopmental Perspective of Surgical Necrotizing Enterocolitis: The Role of the Gut-Brain Axis," Mediators of Inflammation, vol. 2018, Article ID 7456857, 8 pages, 2018.

2. Hou, Y., Lu, X., \& Zhang, Y. (2018). "IRAK Inhibitor Protects the Intestinal Tract of Necrotizing Enterocolitis by Inhibiting the Toll-Like Receptor (TLR) Inflammatory Signaling Pathway in Rats." Medical Science Monitor: International Medical Journal of Experimental and Clinical Research, 24, 3366-3373.

3. Josef Neu, M.D., and W. Allan Walker, M.D. (2011). "Necrotizing Enterocolitis." N Engl J Med 2011;364:255-64.

4. Katherine E. Gregory, Christine E. DeForge, Kristan M. Natale, Michele Phillips, Linda J. Van Marter. "Necrotizing Enterocolitis in the Premature Infant. Neonatal Nursing Assessment, Disease Pathogenesis, and Clinical Presentation." Advances in Neonatal Care. Vol. 11, No. 3, pp. 155-164

5. Hodzic Z, Bolock AM and Good M (2017) The Role of Mucosal Immunity in the Pathogenesis of Necrotizing Enterocolitis. Front. Pediatr. 5:40.

6. Ledesma-Colunga, M. G., Adán, N., Ortiz, G., Solís-Gutiérrez, M., López-Barrera, F., Martínez de la Escalera, G., \& Clapp, C. (2017). Prolactin blocks the expression of receptor activator of nuclear factor $\kappa \mathrm{B}$ ligand and reduces osteoclastogenesis and bone loss in murine inflammatory arthritis. Arthritis Research \& Therapy, pp. 19, 93.

7. Kyuwhan Jung, Jeong-Hyun Kim, Hyun Sub Cheong, Eun Shin, Seong-Ho Kim, Ji-Yeon Hwang, Eunyoung Lee, Mi-Ok Yoon, Seong-Hee Kim, Cherry Ann Sio, Hyoung Doo Shin, Sung-Eun Jung. (2015) "Gene expression profile of necrotizing enterocolitis model in neonatal mice." International Journal of Surgery, 23, pp. 28-34.

8. Scott M. Tanner, Taylor F. Berryhill, James L. Ellenburg, Tamas Jilling, Dava S. Cleveland, Robin G. Lorenz, Colin A. Martin. (2015). "Pathogenesis of necrotizing Enterocolitis Modeling the Innate Inmune Response." The American Journal of Pathology. Vol. 185, No. 1

9. Van Vollenstee, F. A., Jackson, C., Hoffmann, D., Potgieter, M., Durandt, C., \& Pepper, M. S. (2016). Human adipose derived mesenchymal stromal cells transduced with GFP lentiviral vectors: assessment of immunophenotype and differentiation capacity in vitro. Cytotechnology, 68(5), pp. 2049-2060. 